\title{
The elderly in residential care: mortality in relation to functional capacity
}

\author{
L. J. DONALDSON, D G CLAYTON, AND MICHAEL CLARKE \\ From the Department of Community Health, School of Medicine, University of Leicester
}

SUMMARY A census in which 4514 people aged 65 and over had been enumerated in all types of $\frac{\overline{\bar{c}}}{\sqrt{5}}$ institutional care both within and outside the National Health Service in Leicestershire was taken as a starting point for the present investigation. This entire population was followed up for one year to determine its mortality experience. Mortality was described by three measures: (a) the $\vec{\circ}$ proportion surviving for one year from the date of the census, (b) the standardised mortality ratio $\rightarrow$ (using the population of Leicestershire in 1977 as a standard), and (c) using a life-table analysis, the $\vec{\sigma}$ percentage survival to specified time periods after admission to institutional care. We discuss the $\frac{\Omega}{\infty}$ relationship of these indices to functional capacity, indicated by the ability to undertake basic? activities of daily living (ADL), and to type of institution.

The extent to which an individual can easily undertake the basic activities of daily living in many cases determines his degree of independence within the community. A recent census of all people aged 65 and over in the spectrum of institutional care provided both within and outside the National Health Service in Leicestershire ${ }^{1}$ has identified patterns of disability in each type of facility. This paper examines the mortality of that population during the first year after the census, both for differing levels of disability and for those in different types of institutional care.

\section{Method}

At the end of 1976, a one-day census was carried out to enumerate all people aged 65 and over who were resident in any form of institution provided by the National Health Service, the local authority social service department, or voluntary and private bodies in Leicestershire. The results of this survey have been described elsewhere. ${ }^{1}$ It undertook to record, among other things, the personal characteristics of each elderly person and to measure the extent to which basic activities of daily living were performed. The assessment of activities of daily living was based on a modified version of a scale originally validated in a population of chronic schizophrenics. ${ }^{2}$ It was later used, and further validated, in a mixed population of schizophrenics and patients with other long-standing disorders $^{3}$ and, most recently, in a population of more

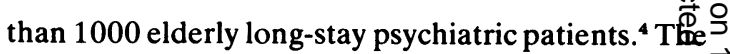
staff immediately concerned with looking after te elderly persons were asked to complete ga questionnaire (Table 1), in which they had to pici statement from each of five categories which mot $\overrightarrow{0}$ nearly described that person's capacities. Withnin each category, points were allotted to each statement and, by addition, a total activities of daily living (ADL) score was obtained in each case. On this scale, the maximum score of 11 indicated relatively high dependency and the minimum score of 0 relative $\bar{D}$ independence. One year after the date of the original $\stackrel{2}{\vec{a}}$ census, a follow-up was undertaken of the 4514 을 elderly people (who had originally been enumerated) to determine which of them had died during the ensuing 12 months. This was performed by obtaining

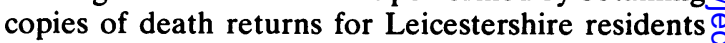
held by Leicestershire Area Health Authority and? searching through them for evidence of a death in a 3 member of the institutional population in the census. Details were then recorded of date and cause of $O$ death.

\section{Results}

One thousand one hundred and one of the $4514 \pi$ people counted in the census were dead one year after the census date. In the original survey, no 0 assessment of ADL was asked for on patients who $\mathrm{W}$ had been admitted less than 28 days before the census date; it was reasoned that, in theseco 
Table 1 Questionnaire about activities of daily living $(A D L)$, with the score for each statement or answer

To indicate the correct answer to a question, tick the relevant box. Please tick only one answer for each question. If there are difficulties in answering these questions, for example, patient in a coma, please complete as many as possible and comment below as necessary

\section{MOBILITY}

Bedfast $\square 3$ Mobile with attendant or mechanical aid $\square 2$ Ambulant apart from stairs $\square 1 \quad$ Fully ambulant $\square 0$

2 CONTINENCE OF URINE

Was frequently incontinent during the week $\square 3$ Was incontinent at least once during the week $\square 2$ Needed raising at night or sending to the lavatory during the day in case of incontinence, but was not actually incontinent when this was done $\square 1$ Needed no raising or sending and was not incontinent $\square 0$

\section{FAECAL INCONTINENCE $\quad$ YES $\square 1 \quad$ NO $\square 0$}

4 WASHING-DRESSING

Was shaved, washed or dressed at least once $\square 2$ Could shave, wash and dress but was supervised $\square 1$ Had no supervision $\square 0$

\section{FEEDING}

Was spoon fed at least once during the week $\square 2$ Was not spoon fed but required supervision $\square 1$ Was neither spoon fed nor supervised $\square 0$

circumstances, staff would not have had sufficient opportunity to gain a firm impression of the functional capacity of the patient in their care. In this section, therefore, results analysed by ADL score refer only to the 3561 patients admitted to the respective type of institution 28 days or more before the census date.

Table 2 shows that the overall one-year survival figure was $77 \%$ and when ADL score was taken into consideration much worse survival figures were observed for the higher dependency groups. The effect of age on the same index is shown in Table 3.

Table 2 Effect of different activities of daily living ( $A D L$ ) scores on per cent survival at one year.

\begin{tabular}{lcl}
\hline ADL score & Nos. & \% survival at one year \\
\hline 0 & 795 & 89 \\
$1-2$ & 873 & 84 \\
$3-4$ & 599 & 77 \\
$5-6$ & 445 & 75 \\
$7-8$ & 367 & 65 \\
$9-11$ & 482 & 57 \\
Total & 3561 & 77 \\
\hline
\end{tabular}

The proportion of survivors is greatest in the youngest $(83.4 \%)$ and least in the oldest $(71.2 \%)$ age groups, although the effect was not so marked as that between lowest and highest ADL scores. Table 3 also shows that the fall in survival with increasing age was seen in the groups with the lower ADL scores, but no such trend was observed in the ADL score range $7-11$. When one-year survival was analysed by place of residence (Table 4), the lowest figures were observed for people in geriatric wards $(65.0 \%)$ and acute wards $(68.3 \%)$ at the time of the census. The other populations (those in psychiatric hospitals, homes for the elderly, homes for the handicapped, and private nursing homes) fared better, with one-year survival about $80 \%$. In each type of institution, a fall in survival was observed between those with the lowest and those with the highest ADL scores. The effect was not so clear-cut, however, in the populations of the acute and geriatric wards, where lower one-year survival was observed in the ADL score band 0-2 compared with the same dependency group in other types of institution. However, when cancer deaths were excluded from

Table 3 Effect of age and activities of daily living ( $A D L)$ score on per cent survival at one year

\begin{tabular}{|c|c|c|c|c|c|c|c|c|c|c|}
\hline \multirow[b]{2}{*}{$A D L$ score } & \multicolumn{2}{|c|}{$\begin{array}{l}\text { Age group (years) } \\
65-74\end{array}$} & \multicolumn{2}{|c|}{$75-84$} & \multicolumn{2}{|c|}{$85-94$} & \multicolumn{2}{|l|}{$95+$} & \multicolumn{2}{|c|}{$A L L A G E S$} \\
\hline & No. & \% survival & No. & \% survival & No. & \% survival & No. & \% survival & No. & \% survival \\
\hline $0-2$ & 382 & $90 \cdot 1$ & 720 & $86 \cdot 5$ & 505 & $84 \cdot 4$ & 49 & $81 \cdot 6$ & 1656 & 86.5 \\
\hline $3-6$ & 163 & $85 \cdot 9$ & 407 & $75 \cdot 4$ & 407 & $75 \cdot 0$ & 57 & $68 \cdot 4$ & 1034 & 76.5 \\
\hline $7-11$ & 140 & $62 \cdot 1$ & 330 & $61 \cdot 8$ & 315 & $57 \cdot 8$ & 57 & $64 \cdot 9$ & 842 & $60 \cdot 6$ \\
\hline Total* & 685 & $83 \cdot 4$ & 1457 & $77 \cdot 8$ & 1227 & 74.4 & 163 & $71 \cdot 2$ & 3532 & $77 \cdot 4$ \\
\hline
\end{tabular}

- Total differs from that in other tables because ages of 29 patients were not recorded. 
L. J. Donaldson, D. G. Clayton, and Michael Clarke

the analysis, part of the difference disappeared. Patients with malignant disease, although remaining relatively independent, may deteriorate and die quite rapidly.

In addition to calculations of one-year survival, the mortality experience of the institutional population was compared with that of a standard population. The application of age-specific death rates for Leicestershire in 1977 (standard population) to the institutional population yielded an 'expected' number of deaths among the institutional elderly. Figures 1 and 2 show the results of this analysis. The ratio of 'observed' to 'expected' deaths has been expressed as a percentage to produce a standardised mortality ratio (SMR). As might be expected, the institutional elderly population had an unfavourable mortality experience compared to the standard population (Fig. 1) as evidenced by the SMR of 225 .

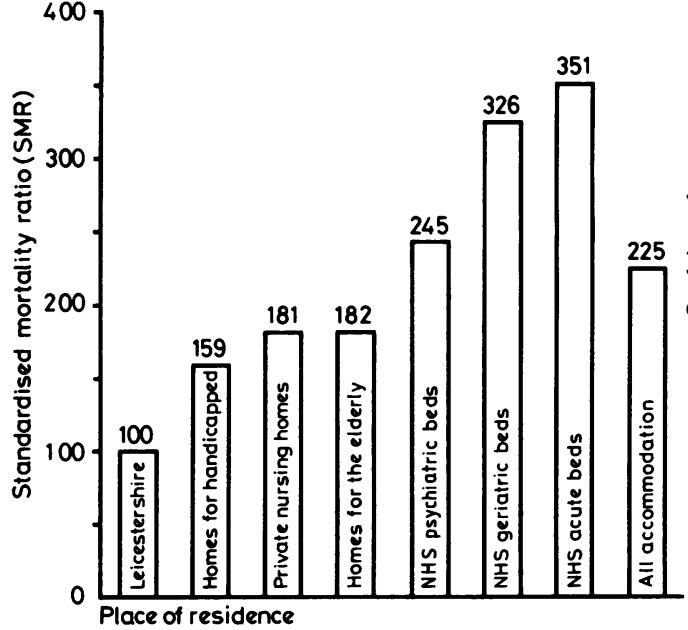

Fig. 1 SMRs for elderly populations in each type of accommodation, using Leicestershire 1977 as standard population.

Within the different types of institution, the three NHS facilities-acute, geriatric, and psychiatric hospitals - fared worse than the non-NHS facilities, although the latter group still experienced markedly higher mortality than the general population. Comparison for different ADL scores (Fig. 2) showed that the groups with lowest scores had an SMR only slightly greater than 100 and that it reached very high values for the groups with the highest ADL scores. 


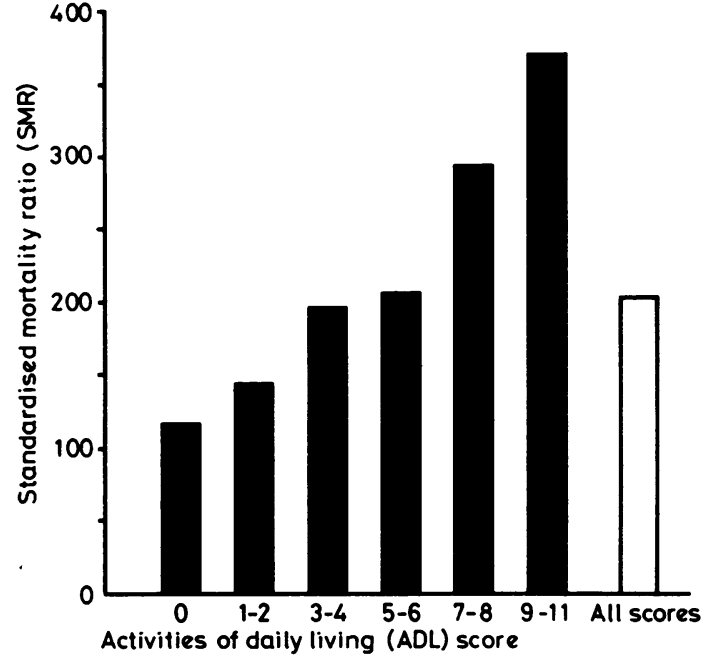

Fig. 2 SMRs for activities of daily living $(A D L)$ score ranges, using Leicestershire 1977 as standard population.

Finally, the mortality data were analysed using a standard life-table technique: estimates were made of the survival to various time points after admission to each type of institution (Fig. 3) and for each group of ADL scores (Fig. 4). It should be noted that survival estimates for various ADL scores were not made beyond two years because patients were assessed at the time of the census, not on admission. The results presented in Fig. 3 show that after admission to any type of institution only about half of

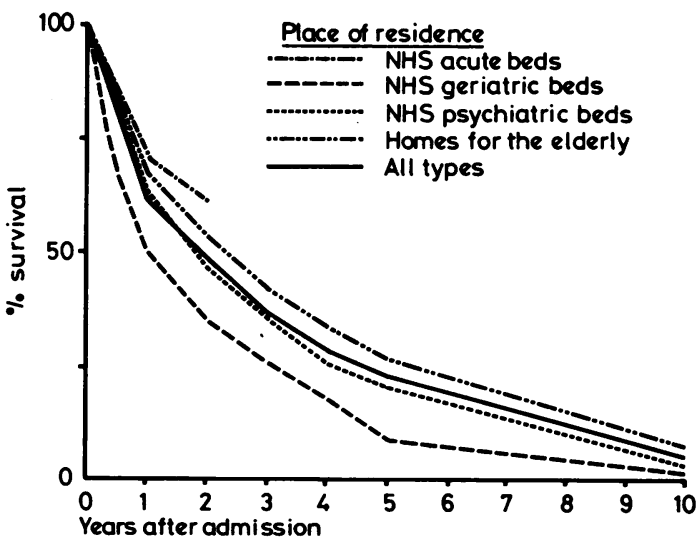

Fig. 3 Estimated per cent survival to specified time periods after admission to different types of accommodation.

Data for patients in homes for the handicapped and private nursing homes have been excluded because of small numbers. all elderly people would be expected to survive for two years, and only a quarter for as long as five years. The geriatric hospital population would be expected to do worst, with about a third surviving for two years and a tenth for five years after admission. The predicted outlook for the population in homes for the elderly is better, but even here just under half would be dead two years after admission. Predicted survival by ADL score (Fig. 4 ) shows that only $21 \%$ in the highest ADL score group would survive for two years after admission.

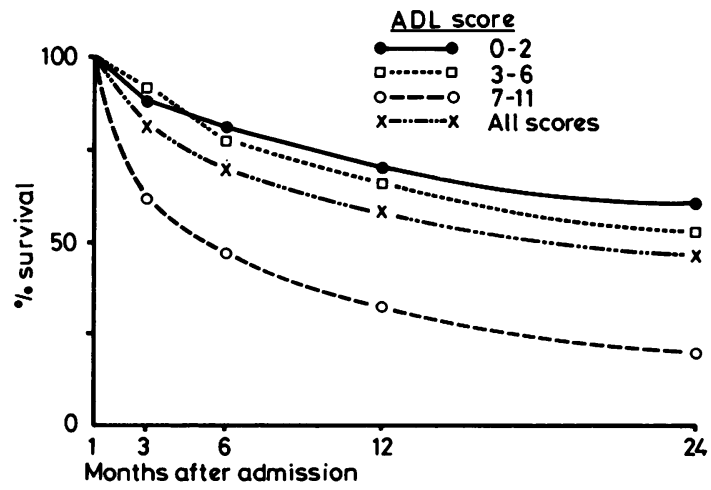

Fig. 4 Estimated per cent survival to specified time periods after admission by different activities of daily living ( $A D L)$ scores.

\section{Discussion}

Studies of mortality of the elderly in institutional care have tended to concentrate on one type of institution. For example, Brody 5 found an overall one-year mortality of $27 \%$ in a population of mentally impaired institutional elderly, which is higher than the $18.4 \%$ for elderly people in psychiatric hospitals noted in our study but not as great as the mortality of $35 \%$ observed for geriatric hospital patients. Lieberman ${ }^{6}$ found a first-year mortality of $24.7 \%$ in a home for the Jewish aged in Chicago, and Smith and Lowther ${ }^{7}$ observed a mortality of $27 \%$ in a local authority residential home in Edinburgh. Both of these studies, however, measured mortality for new admissions to the institutions in question; thus their one-year mortality figures are more appropriately compared to the values obtained by the life-table analysis in our study. Such a comparison reveals that the estimated one-year mortality for residents in homes for the elderly in Leicestershire was $32 \cdot 8 \%$, considerably higher than the figures obtained by those other workers. However, studies of mortality in single institutions give results from which it may not be valid to draw general conclusions. 
Goldfarb et $a l^{8}$ drew a sample of elderly people from a representative group of voluntary homes, proprietary nursing homes, and State hospitals in New York City. They found an overall one-year mortality of $24 \%$ compared with $22.8 \%$ for all the types of institution surveyed in our study. The same workers found that mortality within one year was higher in the State hospitals $(37 \%)$ than in old age homes $(18 \%)$. These mortality figures at one year are similar to those observed for the geriatric hospital (35\%) and homes for the elderly $(20.7 \%)$ in the Leicestershire institutional elderly population (Table 3). However, such international comparisons are made difficult by the different types of care provided for elderly people in different countries. Our study minimises the problem of selection by taking as its starting point all those over 65 resident in any type of institutional care in a defined geographical area at a point in time. Each category of care thus represents a grouping of a number of individual institutions.

Assessment of elderly patients on the basis of their medical diagnoses alone is of little use to those concerned with providing services. It is often impossible to deduce a principal problem amid diffuse and multiple pathologies. An assessment based on levels of function is appealing in that it gives a common currency through which degree of impairment can be matched to type of care. A number of studies have examined the way in which disability in institutionalised elderly people, expressed in various ways and of differing levels of severity, influences mortality. The highest mortality in institutional populations of the elderly is associated with poor mental status, high physical dependence, loss of mobility, and incontinence of urine or faeces. ${ }^{910}$ Brauer et al, ${ }^{9}$ in a study of a large geriatric institution in Copenhagen, calculated a probability of death in one year of about $13 \%$ for those patients classified as 'independent' with respect to their need for nursing care. Figures of $34.1 \%$ (female) and $46.7 \%$ (male) were described for those patients judged to be 'heavily dependent'. Goldfarb, ${ }^{10}$ using the sample of institutionalised elderly patients cited earlier, found a one-year mortality of $15 \%$ for patients with 'mild' physical dependency and $37 \%$ for those with 'severe' physical dependency. Functional assessments, which include some of the above measures with other necessary components of normal activity such as washing, dressing, and feeding, provide a more practical guide to the type of care required by an individual patient. Such an index of activities of daily living (ADL) has been developed and validated by Katz and his colleagues. ${ }^{11}$ The application of this index in a recent study of the elderly in nursing homes in the United States $^{12}$ has given probabilities of survival to specified time periods after admission. The probabilities of survival to six months, one year, and two years after admission in the lowest dependency group were thus found to be $0 \cdot 847,0.741$, and 0.599 respectively. The estimated survival to the same time periods after admission in the Leicestershire population within the ADL score range $0-2$ were $0 \cdot 823,0 \cdot 725$, and $0 \cdot 614$. $\frac{T}{\mathbb{D}}$ When the same index at the same time periods after admission is compared for the highest dependency group in the American nursing home population and Leicestershire patients in the ADL score range 9-11, the following results are obtained (American study figure shown first, Leicestershire figure in brackets): six months: $0.498(0.485)$; one year: $0.401(0.342)$; two years: $0.275(0.207)$. Thus, although the ADL score used in our study has not been validated के previously in all the settings in which it has been used, $\overrightarrow{0}$ it appears to correlate with mortality in the expected direction and with similar scores validated by other workers.

The predicted increase in the numbers of people aged 65 and over in the next few decades will produce the greatest increase in the very old. It is in thes oldest age groups where dependency levels are highest and thus higher proportions of the populatio will require some form of institutional care. ${ }^{1} \mathrm{Th}$ modern approach to providing services for the elderly is to create a spectrum of care through which $\subseteq$ the needs of each elderly person are matched to the $\overline{0}$ most appropriate form of care early in the course of $\overrightarrow{0}$ their illness; moreover, should they deteriorate of improve, then they may be rapidly transferred to $\vec{a}$ setting more suited to their altered needs. Within this spectrum of care the level of staff and resources differs markedly. Our study has shown that a substantial proportion of elderly people with high levels of disability are cared for in homes outside the NHS and that, because of the strong correlation between high ADL score and high mortality score, staff with little or no formal nursing training may therefore be severely extended in dealing with high dependency patients, with relatively little pay-off. The development of a functional assessment similar to the one described here has several attractions. Firstly, it conveniently summarises the disability resulting from a wide variety of underlying pathological processes in a way which is useful to and easily understood by staff in a range of modes of care. 우 Thus it may facilitate the initial placement of patients $D$ in appropriate accommodation and may suggest the need for their movement within the system when $N$ necessary. Secondly, it allows a basis for the establishment of overall policy and resource $\tilde{\mathcal{O}}$ allocation in determining what the most appropriate balance of care should be. Lastly, it provides a basis, 을 albeit crude, for the evaluation of the different types 0 
of care by comparison of the deterioration or improvement in dependency and differing mortality experience in different settings.

We thank Mr. John Woods for help with computing and Leicestershire Area Health Authority (Teaching) for financial support.

Reprints from Dr. L. J. Donaldson, Department of Community Health, School of Medicine, University of Leicester, Clinical Sciences Building, Leicester Royal Infirmary, PO Box 65, Leicester LE2 7LX.

\section{Reference}

${ }^{1}$ Clarke $\mathrm{M}$ et al. The elderly in residential care: patterns of disability. Health Trends 1979; 11: 17-20.

${ }^{2}$ Wing JK, Brown GW. Institutionalism and Schizophrenia: A comparative study of three hospitals, 1960-1968. Cambridge: Cambridge University Press, 1970.

${ }^{3}$ Philip AE, McKechnie AA. The assessment of long-stay psychiatric patients. Soc Psychiatry 1969; 4: 66-81.
${ }^{4}$ Clarke M, Waller J, Webster B. The assessment and progress of long-stay and elderly psychiatric patients: the predictive validity of a ward behaviour questionnaire. Br J Psychiatry 1975; 127: 149-56.

${ }^{5}$ Brody EM et al. Predictors of mortality in the mentally impaired institutionalised aged.J Chronic Dis 1972; 25: 611-20.

${ }^{6}$ Lieberman MA. Relationship of mortality rates to entrance to a home for the aged. Geraitrics 1961; 16: 515-9.

${ }^{7}$ Smith RG, Lowther CP. Follow-up study of two hundred admissions to a residential home. Age Ageing 1976; 5: 176-80.

soldfarb AI, Fisch M, Gerber IE. Predictors of mortality in the institutionalised aged. Dis Nerv Syst 1966; 27: 21-9.

${ }^{9}$ Brauer E, Mackeprang B, Bentzon MW. Prognosis of survival in a geriatric population. Scand J Soc Med 1978; 6: $17-24$.

${ }^{10}$ Goldfarb AI. Predicting mortality in the institutionalised aged: a seven-year follow-up. Arch Gen Psychiatry 1969; 21: 172-6.

${ }^{11} \mathrm{Katz} \mathrm{S}$ et al. Studies of illness in the aged. The index of ADL: a standardised measure of biological and psychosocial function. JAMA 1963; 185: 914-9.

12 Jones EW, Densen PM, McNitt BJ. Assessing the quality of long-term care. Research Summary Series. Maryland: National Centre for Health Services Research, 1978. 